\title{
Atitude implícita e explícita em relação à obesidade entre treinadores de futsal da cidade de Londrina - PR
}

\author{
Implicit and explicit attitude regarding the obesity among \\ coaches of futsal schools of the city of Londrina - PR
}

\section{Actitud implícita y explícita en cuanto a la obesidad entre entrenadores de futsal de la ciudad del Londrina - PR}

\author{
iD (9) Lucas Rafael De Castro Bettone \\ Universidade Estadual de Londrina, Londrina, Paraná, Brasil \\ e-mail: lucasbettone@gmail.com \\ iD Márcia Greguol \\ Universidade Estadual de Londrina, Londrina, Paraná, Brasil \\ e-mail:mgreguol@uel.br
}

Resumo: Este estudo objetivou investigar a atitude implícita e explícita de 15 treinadores nas escolas de futsal da cidade de Londrina-PR em relação à obesidade. Foi utilizado o Teste de Associação Implícita para avaliar o preconceito implícito e o Questionário Crandall para o preconceito explícito em relação à obesidade de crianças praticantes de futsal da cidade. Os resultados apontaram uma maior Antipatia ao obeso por parte dos treinadores eutróficos. Ainda, os treinadores mais jovens apresentaram atitudes explícitas mais negativas em relação à obesidade, associando-a à "burrice" ou "preguiça". Destaca-se que as atitudes implícitas negativas devem ser reduzidas, pois têm potencial de causar graves problemas às crianças e que os treinadores têm papel fundamental nesse processo, visto que as atitudes negativas podem afastar as crianças da prática esportiva.

Palavras-chave: Atitude implícita. Obesidade. Futsal. Obesidade infantil. 
Abstract: This study aimed to investigate the implicit and explicit attitude towards obesity in 15 coaches in the futsal schools of the city of Londrina-PR. The "Implicit Association Test" was used to evaluate the implicit prejudice and the "Crandall" Questionnaire for the explicit prejudice in relation to obesity in futsal children of the city of Londrina. The results showed a greater Antipathy to the obese for the eutrophic trainers. In addition, the younger trainers presented more negative explicit attitudes towards obesity, associating obesity with "stupidity" or "laziness". It is noteworthy that the implicit negative attitudes should be reduced, because they have the potential to bring serious problems to children and that the coaches have a fundamental role in this process, since negative attitudes can lead children away from sports practice.

Keywords: Implicit attitudes. Obesity. Initiation. Futsal. Child obesity.

Resumén: El objetivo de este estudio era investigar la actitud implícita y explícita hacia la obesidad en 15 entrenadores de las escuelas de futsal de la ciudad de Londrina-PR. Se utilizó el "Test de asociación implícita" para evaluar el prejuicio implícito y el cuestionario "Crandall" para el prejuicio explícito en relación con la obesidad en niños de futsal de la ciudad de Londrina. Los resultados mostraron una mayor antipatía hacia los obesos para los entrenadores eutróficos. Además, los entrenadores más jóvenes presentaron actitudes explícitas más negativas hacia la obesidad, asociando la obesidad con "estupidez" o "pereza". Cabe destacar que las actitudes negativas implícitas deben reducirse, ya que tienen el potencial de causar graves problemas a los niños y que los entrenadores tienen un papel fundamental en este proceso, ya que las actitudes negativas pueden alejar a los niños de la práctica deportiva.

Actitud implícita. Obesidad. Futsal. Obesidad infantil.

Submetido em: 14-02-2019

Aceito em: 05-09-2019 
Atitude implícita e explícita em relação à obesidade entre treinadores de futsal... Lucas Rafael De Castro Bettone • Márcia Greguol

\section{Introdução}

A obesidade já é considerada uma pandemia mundial. Políticas de saúde pública em diversos países foram criadas a fim de resolver esse problema que vem crescendo com o decorrer dos anos e que atinge a todos os segmentos populacionais, independente de raça, gênero, nível socioeconômico ou faixa etária. O sobrepeso e a obesidade infantil têm recebido especial atenção pelas políticas de saúde públicas e profissionais atuantes, visto que mais de 170 milhões de crianças no mundo todo se encontram nessa situação (SWINBURN et al., 2011).

Entre as principais causas do sobrepeso e da obesidade infantil destacam-se a alimentação inadequada e o sedentarismo. A ingestão de frituras, alimentos e bebidas industrializados, e a não ingestão de alimentos saudáveis como frutas e hortaliças aumentam em até cinco vezes a chance de as crianças tornarem-se obesas (GIUGLIANI; TRISCHE, 2005; MONDINI et al., 2007). Outro motivo importante para o aumento do sobrepeso e da obesidade infantil foi a mudança causada pela revolução tecnológica. Os jogos eletrônicos, computadores, tablets e smartphones acarretaram numa diminuição da atividade física, agravando ainda mais a tendência ao sedentarismo (TEMPLE et al., 2007).

Crianças obesas são estigmatizadas por professores e colegas e isso reflete em seus desempenhos escolares (PUHL; HEUER, 2009). Segundo pesquisas, estudantes obesos obtêm menores notas nos níveis escolares básicos. Esses dados levam a concluir que as notas mais baixas podem ser devido a um tratamento diferente recebido pelas mesmas por parte dos professores e colegas (MACCANN; ROBERTS, 2013). O estigma para com a criança obesa também está diretamente relacionado ao sedentarismo, visto que crianças que são alvos de preconceito e zombarias, tanto por parte de colegas quanto de professores, tendem a diminuir seu prazer pela atividade física e sua participação no esporte, preferindo to- 
Atitude implícita e explícita em relação à obesidade entre treinadores de futsal... Lucas Rafael De Castro Bettone • Márcia Greguol

mar parte em atividades sedentárias (LYNAGH; CLIFF; MORGAN, 2015).

Algumas das consequências sociais que a criança obesa vivencia devido ao preconceito é o isolamento, a discriminação e o bullying escolar, condições estritamente relacionadas entre si e com consequências psicológicas. Ressalta-se que o bullying escolar e a discriminação levam a criança a querer isolar-se, agravando a ansiedade e gerando perda da autoestima (LEÃO, 2010).

Logo, o prejuízo que o estigma antiobesidade traz às crianças obesas é gigantesco e muitas vezes irreversível. E é responsabilidade, também, dos professores e treinadores conscientizar as crianças e educá-las a fim de que compreendam que todas as crianças têm direito às mesmas possibilidades e que, mais importante, deve haver o respeito pelos sentimentos, valores e princípios, e não o foco no seu peso ou qualquer característica externa (LOSADA; RIJAVEC, 2017).

Uma boa iniciação esportiva é fundamental para que a criança se interesse pelo esporte. A prioridade do esporte e de quem o ensina deve ser formar um ser humano autônomo e participante, eliminando discriminações e preconceitos. Para Santana (2002, p. 1), "a iniciação esportiva é um fenômeno humano absolutamente complexo", então dimensões humanas sensíveis como a sociabilidade, moralidade e a amabilidade não deveriam ser menosprezadas. Logo, o objetivo das escolas de iniciação esportiva deve ser proporcionar o aprendizado da modalidade, mas não o aprendizado técnico como fim e sim como um meio para vivências de outras várias possibilidades intelectuais, educacionais, motoras e, também, esportivas. O simples aprendizado técnico da modalidade na iniciação esportiva privilegiaria somente alguns, não se importando devidamente com os outros que, por muitas vezes, são vítimas de atitudes negativas de treinadores, acarretando num isolamento (ou autoisolamento) e abandono da prática esportiva (RAMOS; NEVES, 2008). Desta forma, o fato de um treinador esportivo discriminar uma criança pela sua obesidade pode ser um fator que acarrete seu abandono precoce da modalidade e, por este motivo, 
Atitude implícita e explícita em relação à obesidade entre treinadores de futsal... Lucas Rafael De Castro Bettone • Márcia Greguol

torna-se relevante conhecer as atitudes dos profissionais diante de um aluno nesta condição.

Assim, tendo em vista a relevância do tema, este estudo teve como objetivo investigar a atitude implícita e explícita em treinadores nas escolas de futsal da cidade de Londrina em relação a crianças obesas, além de verificar possíveis relações destas atitudes com o índice de massa corporal, a idade, o sexo e o tempo de experiência do treinador. A pesquisa tem por intuito verificar se existe, por parte dos treinadores, preconceito implícito ou explícito com relação aos alunos obesos que frequentem as aulas de futsal.

\section{Metodologia}

Tipo de pesquisa e número da amostra

Esse é um estudo do tipo descritivo, com delineamento transversal. Participaram do estudo 15 treinadores, sendo uma do sexo feminino e 14 do sexo masculino, de escolas de futsal de Londrina, correspondendo a $75 \%$ do total de treinadores da cidade que atuavam em equipes com crianças dos 4 aos 11 anos. Os treinadores de equipes participantes da Liga Metropolitana de Futsal foram contatados via celular e, os que aceitaram o convite, assinaram um termo de consentimento livre e esclarecido para participar da pesquisa. Para a participação no estudo, os treinadores deveriam apresentar pelo menos dois anos de experiência profissional. A pesquisa obteve aprovação no Comitê de Ética em Pesquisa com Seres Humanos da Universidade Estadual de Londrina, sob parecer número 2.323.254. 
Atitude implícita e explícita em relação à obesidade entre treinadores de futsal... Lucas Rafael De Castro Bettone • Márcia Greguol

\section{Instrumentos e procedimentos}

Foram avaliadas as variáveis massa corporal (utilizando balança digital com precisão de 100g) e estatura (utilizando estadiômetro com precisão de $0,1 \mathrm{~cm}$ ) dos treinadores para posterior cálculo do Índice de Massa Corporal (IMC) por meio da fórmula: massa corporal (em kg) / estatura (em m) ${ }^{2}$.

Para a caracterização socioeconômica dos participantes foi utilizado o Instrumento de Nível socioeconômico da ABEP, que classifica as famílias por renda bruta em estratos A1, A2, B1, B2, C1, C2, D e E. Esse instrumento é composto por uma escala de itens, bens materiais, os quais o respondente assinala a quantidade que possui. Essa quantificação é contada em uma escala de valores e posteriormente classificada nos estratos já definidos.

Para avaliar as atitudes implícitas dos treinadores de Futsal com relação à obesidade, foi utilizado o Teste de Associação Implícita - "Implicit Association Test" (IAT) (GREENWALD; MCGHEE; SCHWARTZ, 1998), que mede a força de associações automáticas entre um construto e certos atributos e, por meio disso, possibilita avaliar preconceitos que uma pessoa possa estar ignorando ou não querendo relatar.

Foi utilizada a versão impressa do instrumento, sendo que a tarefa dos participantes consistiu em categorizar os itens apresentados (coluna do meio) o mais rapidamente possível, classificando-os nas categorias supraordenadas, alvo ("Pessoas Obesas e Pessoas Magras") e atributiva ("Motivado" e "Preguiçoso", por exemplo). Foram apresentadas duas tarefas de categorização, para cada tarefa foi apresentado também o acoplamento inverso das categorias e os participantes tiveram 30 segundos para realizar cada uma das tarefas. Cada teste foi formulado em uma página estruturada com uma tabela de três colunas. Na coluna central foram apresentados os atributos a serem classificados e as duas colunas laterais representaram as categorias supraordenadas dos atributos de maneira conectada. 
Atitude implícita e explícita em relação à obesidade entre treinadores de futsal... Lucas Rafael De Castro Bettone • Márcia Greguol

Figura 1 - Implicit Association Test (IAT)

\begin{tabular}{|c|c|c|}
\hline $\begin{array}{c}\text { PESSOAS } \\
\text { OBESAS } \\
\text { MOTIVADO }\end{array}$ & $\begin{array}{c}\text { PESSOAS } \\
\text { MAGRAS } \\
\text { PREGUIÇOSO }\end{array}$ \\
\hline & Lento & \\
\hline & Fino & \\
\hline & Determinado & \\
\hline & Obeso & \\
\hline & Preguiçoso & \\
\hline & Magro & \\
\hline & Entusiasmado & \\
\hline & Grosso & \\
\hline & Indolente & \\
\hline & Obeso & \\
\hline & Motivado & \\
\hline
\end{tabular}

\begin{tabular}{|c|c|c|}
\hline $\begin{array}{c}\text { PESSOAS } \\
\text { MAGRAS } \\
\text { MOTIVADO }\end{array}$ & PESSOAS \\
\hline & Obeso & \\
PRESAS \\
\hline & Indolente & \\
\hline & Magro & \\
\hline & Entusiasmado & \\
\hline & Grosso & \\
\hline & Preguiçoso & \\
\hline & Obeso & \\
\hline & Motivado & \\
\hline & Fino & \\
\hline & Determinado & \\
\hline & Magricela & \\
\hline
\end{tabular}

Em relação à avaliação do preconceito explícito, foi utilizado o Questionário Crandall (CRANDALL, 1994) que possui 13 afirmações nas quais os participantes do estudo classificaram cada uma delas com uma pontuação de 1 a 9, sendo que 1 corresponde a "em fortíssimo desacordo" e 9 a "em fortíssimo acordo". O resultado foi calculado para três subescalas:

- "Antipatia" (preconceito explícito contra pessoas obesas);

- "Medo de obesidade" (preocupação pessoal em relação ao peso corporal); 
Atitude implícita e explícita em relação à obesidade entre treinadores de futsal... Lucas Rafael De Castro Bettone • Márcia Greguol

- "Força de vontade" (julga ser a obesidade uma matéria de controle pessoal).

A subescala "antipatia" (itens 1 a 7 e pontuação de 7 a 63) avalia a aversão de uma pessoa no que diz respeito à obesidade; a subescala "medo da obesidade" (itens 8 a 10 e pontuação de 3 a 27) identifica o nível de preocupação de uma pessoa em ganhar peso; já a "força de vontade" (itens 11 a 13 e pontuação de 3 a 27) revela quanta importância se dá à crença de que a obesidade seja um problema de falta de controle pessoal, quer dizer, que seja fruto dos próprios erros da pessoa. A pontuação total pode variar de 13 a 117. Em todas as situações, uma maior pontuação corresponde à maior presença de estigma antiobesidade.

Foram escolhidas escolas de futsal federadas à Liga Metropolitana de Futsal de Londrina. Os coordenadores de cada escola foram contatados e uma reunião foi agendada com os treinadores para consentimento sobre a pesquisa. O questionário foi entregue, coletado e o teste aplicado no local do treino.

\section{Análise estatística}

Os dados foram analisados inicialmente por estatística descritiva, com valores médios e de variabilidade para as variáveis contínuas e porcentagem de respostas para as variáveis categóricas. Para verificar as correlações entre as variáveis de estudo, foi utilizado o teste de correlação de Pearson. As comparações entre os sexos foram realizadas por meio do teste t-student para amostras independentes. Em todos os casos foi adotada significância $p \leq 0,05$. Os dados foram tabulados e analisados no programa estatístico SPSS 24.0. 
Atitude implícita e explícita em relação à obesidade entre treinadores de futsal... Lucas Rafael De Castro Bettone • Márcia Greguol

\section{Resultados}

A Tabela 1 a seguir descreve os dados dos participantes com relação ao sexo, formação inicial, classe econômica e classificação quanto ao IMC (normal ou sobrepeso). Já a Tabela 2 apresenta os valores de média e desvio padrão das variáveis.

Tabela 1 - Tabela descritiva com a frequência das respostas

\begin{tabular}{llll}
\hline Sexo & MASCULINO & FEMININO & \\
& $93,3 \%(n=14)$ & $6,6 \%(n=1)$ & \\
\hline Formação & Ed. Física & Esporte & \\
& $86,7 \%(n=13)$ & $13,3 \%(n=2)$ & \\
\hline Classe econômica & CLASSE A & CLASSE B & CLASSE C \\
& $6,7 \%(n=1)$ & $80 \%(n=12)$ & $13,3 \%(n=2)$ \\
\hline IMC & Eutrófico & Sobrepeso & \\
& $60 \%(n=9)$ & $40 \%(n=6)$ & \\
\hline
\end{tabular}

Tabela 2 - Tabela descritiva média e desvio padrão das demais variáveis

\begin{tabular}{|c|c|c|c|c|}
\hline & Mínimo & Máximo & Média & Desvio Padrão \\
\hline Idade (anos) & 20 & 49 & 27,67 & 8,63 \\
\hline Atuação (anos) & 1 & 30 & 6,67 & 7,52 \\
\hline Estatura (m) & 1,69 & 1,87 & 1,76 & 0,05 \\
\hline $\mathrm{IMC}\left(\mathrm{kg} \square \mathrm{m}^{2}\right)$ & 17,90 & 29,7 & 24,38 & 3,53 \\
\hline Obeso motivado & 0 & 4 & 1,47 & 1,24 \\
\hline Obeso preguiçoso & 0 & 1 & 0,07 & 0,25 \\
\hline Obeso esperto & 0 & 3 & 1,07 & 0,79 \\
\hline Obeso burro & 0 & 0 & 0,00 & 0,00 \\
\hline Antipatia & 7 & 24 & 13,87 & 5,90 \\
\hline Medo da obesidade & 5 & 25 & 12,93 & 5,93 \\
\hline Força de vontade & 5 & 22 & 14,87 & 5,81 \\
\hline Crandall Total & 1 & 67 & 35,53 & 19,45 \\
\hline
\end{tabular}

Observa-se pelos resultados do IAT que ocorreu um número grande de erros nas variáveis "obeso/motivado" e "obeso/esperto", demonstrando que os treinadores tenderam a associar a obesidade à falta de motivação e à falta de esperteza. Na análise seguinte, quando comparados os indivíduos pela classificação do 
Atitude implícita e explícita em relação à obesidade entre treinadores de futsal... Lucas Rafael De Castro Bettone • Márcia Greguol

IMC, a única diferença observada foi na "Antipatia" ao obeso, sendo que o grupo eutrófico apresentou valores significativamente mais altos do que aqueles com sobrepeso neste item $(16,22+6,2$ dos indivíduos eutróficos contra 10, $33+3,4$ dos indivíduos com sobrepeso, com valor de significância $p=0,03$ ). Nos demais itens, não houve diferença significativa.

Por fim, foram feitas análises de correlação entre a idade, tempo de atuação profissional e as respostas obtidas nos questionários, sendo que, das correlações verificadas, as únicas significativas foram com relação à idade e o IMC [quanto maior a idade, maior o valor de IMC $(r=0,56)]$ e ao tempo de atuação profissional e a antipatia ao obeso [quanto maior o tempo de atuação profissional, menor o valor de antipatia ao obeso $(r=-0,63)]$.

\section{Discussão}

O presente estudo procurou investigar atitudes implícitas e explícitas dos treinadores de futsal, comparando e correlacionando os resultados dos testes com outras variáveis como IMC, nível socioeconômico, idade e tempo de atuação profissional. O preconceito é visto como um dos principais responsáveis pela falta de interesse das crianças pelo esporte e por atividades físicas no geral, pois as mesmas evitam as práticas a fim de escapar de possíveis tratamentos injustos por seus professores e treinadores (LYNAGH; CLIFF; MORGAN, 2015).

Entre os participantes do estudo, houve predomínio de treinadores do sexo masculino, com graduação em Educação Física. Com relação às atitudes implícitas, houve maior ocorrência de erros nas associações "obeso/esperto" e "obeso/motivado", evidenciando que, para os treinadores de futsal, existe uma tendência implícita de associar a obesidade à preguiça e/ou falta de inteligência. Esses resultados também foram observados por Gobbi et al. (2017) que, pesquisando profissionais e estudantes de 
Atitude implícita e explícita em relação à obesidade entre treinadores de futsal... Lucas Rafael De Castro Bettone • Márcia Greguol

Educação Física, também verificaram atitudes implícitas negativas com relação à obesidade. Ao relacionar a obesidade a características negativas, segundo os autores, os profissionais podem, ainda que de maneira inconsciente, levar uma criança a abandonar a prática esportiva ou até a nem mesmo iniciar a sua prática, por receio de se sentir excluída e humilhada.

Comparando os indivíduos pela classificação do IMC, foi observada uma diferença significativa no item "Antipatia" ao obeso, sendo que os treinadores eutróficos apresentaram valores mais altos do que os treinadores com sobrepeso $(16,22+6,2$ dos indivíduos eutróficos contra 10, $33+3,4$ dos indivíduos com sobrepeso, com valor de significância $p=0,03$ ). Isso indica que os treinadores eutróficos deste estudo apresentaram tendência a um preconceito explícito contra pessoas obesas, visto que a subescala "Antipatia" avalia a aversão de uma pessoa no que diz respeito à obesidade (CRANDALL, 1994). Já os treinadores que apresentavam sobrepeso demonstraram uma atitude explícita mais positiva com relação às crianças obesas. Estes resultados são semelhantes aos obtidos no estudo de Obara (2015) que verificou que, entre nutricionistas, os profissionais com IMC mais elevados apresentaram menos atitudes negativas em relação a indivíduos obesos. Desta forma, supõe-se que os profissionais que não apresentam problemas com o peso corporal podem manifestar maior intolerância ao fato de a criança estar em situação de obesidade, demonstrando em seus comportamentos aversão à esta condição.

Um estudo realizado na Inglaterra com professores graduados e não graduados em Educação Física mostrou que, apesar de os participantes tenderem a respostas socialmente desejáveis para não demonstrar preconceito em relação à obesidade quando avaliados de maneira explícita, eles endossam um número de estereótipos negativos quanto às crianças obesas, julgando-as como menos saudáveis e menos satisfeitas consigo mesmas em comparação às crianças não obesas. Além disso, a maioria dos participantes acredita que ser obeso é uma das piores coisas que poderia acontecer na vida de uma criança, evidenciando também uma 
Atitude implícita e explícita em relação à obesidade entre treinadores de futsal... Lucas Rafael De Castro Bettone • Márcia Greguol

tendência ao preconceito com relação a esta condição (LYNAGH; CLIFF; MORGAN, 2015). Esse fator é relevante e incide na questão do trato, olhar e expectativas que o profissional terá para com a criança obesa, uma vez que atitudes negativas por parte do professor e do treinador podem fazer com que a criança não se sinta aceita e pertencente ao grupo, levando ao abandono precoce da modalidade esportiva que pratica (PAINI et al., 2018).

Quando correlacionadas as respostas dos questionários com a idade e o tempo de atuação no futsal, foi visto que, quanto maior o tempo de atuação profissional, menor o valor de antipatia ao obeso $(r=-0,63)$. Esses dados mostraram que, com o passar dos anos e com a maior experiência dos treinadores, menor é o valor da antipatia ao obeso, o que nos leva à premissa de que as atitudes e crenças dos treinadores vão sendo desconstruídas no decorrer dos anos com a vivência prática dos treinamentos e demais experiências que o contexto do futsal proporciona. Um estudo realizado por Schwartz et al. (2003) com profissionais da saúde graduados e não graduados em Educação Física mostrou que, correlacionando a idade com o preconceito apresentado no teste IAT, pessoas mais jovens apresentaram maior nível de preconceito em relação àquelas mais velhas, associando a obesidade à preguiça e à burrice.

Destacam-se ainda no presente estudo os valores elevados observados nas atitudes explícitas para os itens "Força de Vontade" e "Medo da Obesidade", que evidenciam que os treinadores de futsal demonstram receio em tornarem-se obesos e que também atribuem de certa maneira a obesidade das crianças à falta de força de vontade em modificar sua situação. Apesar dos valores elevados, não foi observada correlação significativa entre os itens do questionário, o que leva a crer que o fato de a pessoa se preocupar com o próprio peso não é um fator majoritário para ela não gostar de pessoas obesas (BECK, 2016; CRANDALL, 1994).

Em estudo realizado por Lynagh, Cliff e Morgan (2015) em relação às atitudes explícitas para com crianças obesas, foi visto que os itens "Medo de Obesidade" e "Força de vontade" apresentaram 
Atitude implícita e explícita em relação à obesidade entre treinadores de futsal... Lucas Rafael De Castro Bettone • Márcia Greguol

pontuação significativamente maior do que o item "Antipatia", embora este último também tenha apresentado valor elevado. Ainda, uma correlação foi constatada entre os itens "Força de Vontade" e "Antipatia", indicando que os participantes do estudo tendiam a julgar a obesidade como um problema de controle pessoal, fruto dos próprios erros das pessoas. Infelizmente muitas pessoas atribuem falta de força de vontade à causa da obesidade e, ainda, que ser alvo de piadas é uma motivação para que as pessoas emagreçam (BECK, 2016).

Sabe-se que a prática de bullying contra crianças obesas só compromete ainda mais a situação das mesmas. Um estudo realizado por Alexander (2017) constatou uma relação entre ansiedade, alimentação desenfreada e o medo de se tornar obeso. Logo, embora as pessoas não se tornem obesas devido ao medo de ganharem peso, o efeito do bullying e do preconceito as tornam ainda mais ansiosas, o que pode levá-las a um ciclo vicioso que irá agravar ainda mais sua situação.

Por meio da análise dos dados, foi possível notar algumas atitudes negativas implícitas e explícitas por parte dos treinadores que podem, como consequência, afastar as crianças da prática esportiva. Além do prejuízo direto à sua autoestima, as crianças, para evitarem ser discriminadas e alvo de zombaria por parte dos professores e colegas, acabam optando por atividades sedentárias, muitas vezes onde possam ficar sozinhas, tornando-se ainda mais predispostas à obesidade (LYNAGH; CLIFF; MORGAN, 2015; LOSADA; RIJAVEC, 2017).

O estudo apresentou algumas limitações, como o reduzido número de participantes e a utilização apenas de instrumentos quantitativos, que podem não ser sensíveis para uma compreensão mais abrangente da atitude dos treinadores. No entanto, os dados aqui levantados podem oferecer subsídios que sirvam de alerta a pais e profissionais que atuam na iniciação esportiva, no sentido de que sejam evitadas atitudes negativas e, consequentemente, o afastamento precoce da prática por parte de crianças obesas. 
Atitude implícita e explícita em relação à obesidade entre treinadores de futsal... Lucas Rafael De Castro Bettone • Márcia Greguol

\title{
Conclusão
}

Pelos dados obtidos no presente estudo pode-se concluir que algumas atitudes implícitas dos treinadores de futsal denotam preconceito com relação à criança obesa, associando-a a algumas características negativas como "preguiça" ou "burrice". Já as atitudes explícitas foram mais negativas para os profissionais eutróficos e para aqueles mais jovens.

Os profissionais que atuam nas escolas de esporte são diretamente responsáveis, além do ensino de habilidades técnicas e táticas do futsal, por integrar essas crianças, facilitando seu desenvolvimento sociocultural. Logo, os resultados encontrados, principalmente em relação aos treinadores eutróficos e aos mais jovens, preocupam, pois um preconceito implícito e explícito em relação a crianças obesas pode comprometer a eficácia e a qualidade das escolhas comportamentais e educativas.

Os resultados sugerem que estratégias devam ser adotadas a fim de que essas interações entre professores e alunos não sejam prejudicadas pela presença de alguma atitude negativa. Vimos que, com o passar dos anos, os treinadores tendem a desconstruir o preconceito em relação à obesidade, portanto, a formação dos profissionais deve se preocupar em aproximar o indivíduo da realidade dessas crianças, não entendendo-a apenas como um problema, a fim de que o ambiente do treino e das competições possibilite o desenvolvimento da sociabilidade, moralidade e amabilidade, princípios que serão fundamentais para sua vida.

\section{Referências}

\begin{abstract}
ALEXANDER, Katherine E. Attachment anxiety is associated with a fear of becoming fat, which is mediated by binge eating. PeerJ, London, v. 5, p. e3034, 2017.
\end{abstract}


Atitude implícita e explícita em relação à obesidade entre treinadores de futsal... Lucas Rafael De Castro Bettone • Márcia Greguol

ASSOCIAÇÃO BRASILEIRA DE EMPRESAS DE PESQUISA (ABEP). Critério Brasil. Disponível em: http://www.abep.org/criterio-brasil. Acesso em: 26 jun. 2017.

BECK, Amy R. Psychosocial aspects of obesity. NASN School Nurse, v. 31, n. 1, p. 23-27, 2016.

CRANDALL, Christian S. Prejudice against fat people: ideology and self-interest. Journal of personality and social psychology, Washington, v. 66, n.5, p. 882 - 894, 1994.

GOBBI, Erica et al. Um estudo exploratório da tendência "antiobesidade" entre professores e estudantes de educação física italianos. Movimento, Porto Alegre, v. 23, n. 3, p. 963-974, 2017.

GREENWALD, Anthony G.; MCGHEE, Debbie E.; SCHWARTZ, Jordan LK. Measuring individual differences in implicit cognition: the implicit association test. Journal of personality and social psychology, Washington, v. 74, n. 6, p. 1464-1480, 1998.

LEÃO, Letícia Gabriela Ramos. O fenômeno bullying no ambiente escolar. Revista FACEVV, Vila Velha, v. 4, p. 119-135, 2010.

LOSADA, Analia Veronica; RIJAVEC, María Inés María Inés. Consecuencias psicológicas em niños con obesidade producto de la estigmatización social. Revista Neuronum ISSN: 2422-5193 (En línea), v. 3, n. 2, p. 46-65, 2017.

LYNAGH, Marita; CLIFF, Ken; MORGAN, Philip J. Attitudes and Beliefs of Nonspecialist and Specialist Trainee Health and Physical Education Teachers Toward Obese Children: Evidence for "AntiFat" Bias. Journal of School Health, Ohio, v. 85, n. 9, p. 595-603, 2015.

MACCANN, C.; ROBERTS, R. D. Just as smart but not as successful: obese students obtain lower school grades but equivalent test scores to non-obese students. International Journal of Obesity, v. 37, n. 1, p. 40-46, 2013.

MONDINI, Lenise et al. Overweight, obesity and associated factors in first grade school children in a city of the metropolitan region 
Atitude implícita e explícita em relação à obesidade entre treinadores de futsal... Lucas Rafael De Castro Bettone • Márcia Greguol

of São Paulo, Brazil. Cadernos de Saúde Pública, Rio de Janeiro, v. 23, n. 8, p. 1825-1834, 2007.

OBARA, Angelica Almeida. Atitudes de estudantes universitários de nutrição em relação aos indivíduos obesos e à obesidade. 2015. Dissertação (Mestrado em Nutrição em Saúde Pública) - Faculdade de Saúde Pública, Universidade de São Paulo, São Paulo, 2015.

PAINI, Leonor Dias et al. Obesidade infantil e práticas de bullying: questões para a formação docente. Educere-Revista da Educação da UNIPAR, Umuarama, v. 18, n. 2, p. 441-457, 2018. PUHL, Rebecca; HEUER, Chelsea. The stigma of obesity: a review and update. Obesity, v. 17, n. 5, p. 941-964, 2009.

RAMOS, Adamilton Mendes; NEVES, Ricardo Lira Rezende. A iniciação esportiva e a especialização precoce à luz da teoria da complexidade-notas introdutórias. Pensar a prática, Goiânia, v. 11, n. 1, p. 1-8, 2008.

SANTANA, Wilton Carlos de. Iniciação esportiva e algumas evidências de complexidade. In: Simpósio de educação física e desportos do sul do Brasil, v. 14, 2002, Ponta Grossa, Anais [...].Ponta Grossa: Universidade Estadual de Ponta Grossa, 2002. p. 176-180. SCHWARTZ, Marlene. et al. Weight bias among health professionals specializing in obesity. Obesity research, Silver Spring, v. 11, n. 9, p.1033-1039, 2003.

SWINBURN, Boyd et al. The global obesity pandemic: shaped by global drivers and local environments. The Lancet, London, v. 378, n. 9793, p. 804-814, 2011.

TEMPLE, Jennifer $L$. et al. Television watching increases motivated responding for food and energy intake in children. The American Journal of Clinical Nutrition, Oxford, v. 85, n. 2, p. 355-361, 2007.

TRICHES, Rozane Márcia; GIUGLIANI, Elsa Regina Justo.

Obesidade, práticas alimentares e conhecimentos de nutrição em 
Atitude implícita e explícita em relação à obesidade entre treinadores de futsal...

Lucas Rafael De Castro Bettone • Márcia Greguol

escolares. Revista de Saúde Pública, São Paulo, v. 39, p. 541-547, 2005.

\section{Aprovação de comitê de ética em pesquisa}

Pesquisa aprovada pelo Comitê de Ética da Universidade Estadual de Londrina. Título: ATITUDE IMPLÍCITA EM RELAÇÃO À OBESIDADE ENTRE TREINADORES DE FUTSAL DA CIDADE DE LONDRINA - PR

\section{Publisher}

Universidade Federal de Goiás. Faculdade de Educação Física e Dança. Publicação no Portal de Periódicos UFG. As ideias expressadas neste artigo são de responsabilidade de seus autores, não representando, necessariamente, a opinião dos editores ou da universidade.

O presente trabalho não contou com apoio financeiro de nenhuma natureza para sua realização. 